NASA Technical Memorandum $105268^{-}$

$$
\begin{aligned}
& 1 N-39 \\
& 86857
\end{aligned}
$$

p.13

\title{
Aeroelastic Stability Analyses of Two Counter Rotating Propfan Designs for a Cruise Missile Model
}

rem

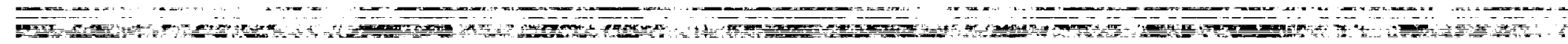

Aparajit J. Māhajan

University of Toledo

$==$ Toledo, Ohio

and

John M, Lucero, Oral Mehmed,

and George L. Stefko

Lewis Research Center

Cleveland, Ohio

April 1992

N/SA

(NASA-TM-105268) AEROELASTIC STABILITY

ANALYSES OF THO COUNTER ROTATING PROPFAN

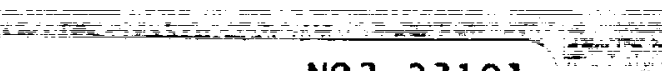

DESIGNS FOR A CRUISE MISSILE MODEL (NASA)

$13 \mathrm{P}$

CSCL $20 K$

N92-23191

Unclas

$63 / 39 \quad 0086857$ 


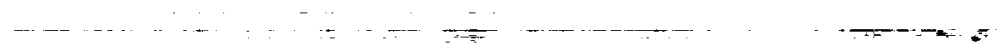




\title{
AEROELASTIC STABILITY ANALYSES OF TWO COUNTER ROTATING PROPFAN DESIGNS FOR A CRUISE MISSILE MODEL.
}

\author{
Aparajit J. Mahajan* \\ University of Toledo \\ Toledo, Ohio 43606 \\ John M. Lucero, Oral Mehmed, and George L. Stefko \\ National Aeronautics and Space Administration \\ Lewis Research Center \\ Cleveland, Ohio 44135
}

\begin{abstract}
Aeroelastic stability analyses were performed to insure structural integrity of two counterrotating propfan blade designs for a NAVY / Air Force / NASA cruise missile model wind tunnel test. This analysis predicted if the propfan designs would be flutter free at the operating conditions of the wind tunnel test. Calculated stability results are presented for the two blade designs with rotational speed and freestream Mach number as parameters. An aeroelastic analysis code ASTROP2 (Aeroelastic STability and Response Of Rropulsion Systems - 2 Dimensional Analysis), developed at LeRC, was used in this project. The aeroelastic analysis is a modal method and uses the combination of a finite element structural model and two dimensional steady and unsteady cascade aerodynamic models. This code was developed to analyze single rotation propfans but was modified and applied to counterrotation propfans for the present work. Modifications were made to transform the geometry and rotation of the aft rotor to the same reference frame as the forward rotor, to input a nonuniform inflow into the rotor being analyzed, and to automatically converge to the least stable aeroelastic mode.

\section{Nomenclature}

[A] generalized aerodynamic matrix
$A_{1}, . ., A_{m}$
torsion contributions of the $m$ normal modes

about the reference axis

[F(t)] nonaerodynamic nodal force vector

$\left\{\mathrm{F}_{0}\right\}$ time-independent nonaerodynamic force vector

[G(t)] generalized motion-independent force vector

$\mathrm{h}$ bending deflection

i $\quad \sqrt{-1}$

j mode index, $j=1,2,3, \ldots, m$

$[\mathrm{K}]$ generalized stiffness matrix

$\left[\mathrm{K}_{\mathbf{g}}^{\mathrm{g}}\right]$

centrifugal softening matrix in physical

coordinates

$[\mathrm{K}(\{\mathrm{u}\})]$ nonlinear stiffness matrix in physical

coordinates

$\mathrm{l}_{11}, . ., \mathrm{l}_{\mathfrak{2}} \quad$ aerodynamic coefficients defined in Ref. 1

$\mathrm{L} \quad$ aerodynamic lift

LCOEF aerodynamic coefficient matrix

m number of normal modes

$\mathrm{M}_{\mathrm{a}} \quad$ aerodynamic moment

[M] physical mass matrix

[M] generalized mass matrix

$\mathrm{N}^{\mathrm{G}} \quad$ number of blades

[P] stiffness matrix defined in Eq. (14)

$\{\mathrm{P}(\{\mathrm{u}\}, \mathrm{t})\}$ aerodynamic nodal force vector

$\left\{\mathrm{P}\left(\left\{\mathrm{u}_{0}\right\}\right)\right\}$ steady-state aerodynamic nodal force vector

[q] vector of generalized coordinates

$\left\{q_{0}\right\} \quad$ amplitude of motion in generalized coordinates

$r \quad$ interblade phase angle index, $r=1,2, \ldots, N$

$\mathrm{s} \quad$ blade length along the reference line

$\mathrm{t}$ time

(u) vector of blade deflections at grid points

$\left\{u_{0}\right\} \quad$ vector of steady state deflections at grid points

[W] modal matrix expressed in terms of individual bending and torsion contributions along the reference line, see Eq. (A3)

$W_{1}, . ., W_{m}$ bending contributions of the $m$ normal modes about the reference axis

$\alpha \quad$ torsional deflection

$\gamma \quad$ eigenvalue defined in Eq. (14)

$[\Delta \mathrm{F}(\mathrm{t})\} \quad$ perturbation nonaerodynamic nodal force vector

$\left\{\Delta \mathbf{P}^{\mathbf{w}}(t)\right\}$ perturbation aerodynamic nodal force vector (motion-independent)

$\{\Delta \mathrm{P}(\{\Delta \dot{\mathrm{u}}\}, \mathrm{t})\}$

perturbation aerodynamic nodal force vector (motion-dependent)

$\{\Delta \mathbf{u}(t)\} \quad$ vector of vibratory deflections at grid points measured relative to $\left\{u_{0}\right\}$

$\zeta_{\mathrm{j}} \quad$ structural damping ratio in $\mathrm{j}$-th mode

$\mu \quad$ real part of $i \sqrt{\gamma}$ (proportional to damping)

$v \quad$ imaginary part of $i \sqrt{\gamma}$ (flutter frequency)

$\sigma_{\mathbf{r}} \quad$ interblade phase angle

[ф] modal matrix

$\omega$ frequency

$\omega_{j} \quad$ frequency of $j$-th mode

\section{Subscripts}

$0 \quad$ steady state value

g generalized (modal)

F values at flutter point

* Resident Research Associate at NASA Lewis Research Center.
\end{abstract}




\section{Superscripts}

differentiation with respect to time
$\mathrm{T}$ transpose

\section{Introduction}

Propfan design and technology have sparked interest in military and civil applications due to the potential for very high fuel efficiency at cruise speeds up to Mach 0.85 . Various studies have been conducted by NASA to evaluate propfan propulsion systems (Refs. 1-3). One can expect from these studies that a counterrotating propfan propulser for cruise missiles offers the potential for significant increases in range or payload compared to current turbojet or turbofan designs. The long-range conventional standoff weapon (LRCSW) program was established as a joint U.S. Navy/Air Force program to develop an advanced, long-range cruise missile powered by a propfan engine. As a part of this program, a joint Navy/Air Force/NASA Propfan Missile Interactions Project was initiated to determine the effects of a propfan engine on missile aerodynamics in a wind tunnel test (Ref. 4). NASA Lewis Research Center (LeRC) designed and fabricated two propfan blade sets for this wind tunnel test. The blade sets were chosen to bracket the various designs proposed by industry for the full-scale propfan missile engines. The major test objective was to investigate structural and aerodynamic interactions between the missile and the propfans. The LeRC team objectives of the program were to design and fabricate two composite counterrotating (CR) propfan blade models which would have high aerodynamic efficiency and structural integrity when mounted on a generic cruise missile model (Ref. 5). The models were to be tested in the NASA Ames Research Center 14 foot wind tunnel. A sketch of the wind tunnel model is shown in Figure 1. Each hub has six blades.

The aerodynamic and acoustic requirements of propfans have resulted in designs with thin, swept, and twisted blades of low aspect ratio and high solidity compared to conventional propellers. Experimental and analytical research has shown that these characteristics of propfans give them the potential to flutter in their operating range (Refs. 1-3). Past theoretical and experimental propfan investigations at LeRC have resulted in various computer codes used to predict aeroelastic stability and response. One such research computer code is ASTROP2 (Aeroelastic STability and Response of Propulsion Systems - 2 Dimensional Analysis). This code was developed to analyze the aeroelastic stability of single rotation propfan blades (Ref. 1). In the present work, it was modified and applied to analyze the counterrotation propfan blade models that were designed for this project.
The objectives of this paper are: (1) to document the results of the aeroelastic stability (flutter) analysis of two CR propfan blade designs developed at LeRC for a cruise missile model, and (2) to describe modifications made to the ASTROP2 code that allowed analysis of the CR rotors and resulted in increased automation and accuracy.

\section{Analytical Formulation}

This formulation is described in detail in Refs. 1 and 6 . A summary is presented here. The aeroelastic equation of motion of a blade can be written as

$$
[M]\{u ̈\}+\left[K_{\mathrm{s}}\right]\{\mathrm{u}]+[\mathrm{K}(\{\mathrm{u}\})]\{\mathrm{u}\}=\{\mathrm{P}(\{\mathrm{u}\}, \mathrm{t})\}+\{\mathrm{F}(\mathrm{t})\}
$$

where $\{u\}$ represents the blade deflections at the grid points, $[M]$ is the mass matrix, $\left[\mathrm{K}_{\mathrm{s}}\right]$ the centrifugal softening matrix, $[K([u])]$ the nonlinear stiffness matrix, $\{\mathrm{P}(\{\mathrm{u}], t)\}$ the equivalent aerodynamic nodal force vector, and $\{F(t)\}$ the equivalent nonaerodynamic force vector. Because of the large steady deflections and the consequent need for the geometric nonlinear theory of elasticity in which the strain and displacement relations are nonlinear, the stiffness matrix $[\mathrm{K}(\{\mathrm{u}\})]$ is a function of nodal displacements and, hence, is nonlinear. This provides the additional geometric differential stiffness due to centrifugal stiffening terms. The displacement dependent centrifugal softening terms are included in the matrix $\left[\mathrm{K}_{b}\right]$. The rotation also introduces Coriolis forces, but these have been shown to be negligible for thin propfan blades in Ref. 7 . Hence, they are not included in the present formulation.

\section{Linearization of Equations}

Equation (1) is generally nonlinear and is valid for calculating performance, stalled and unstalled flutter, forced response, steady-state deflections, frequencies and mode shapes. One solution method is to directly integrate in the time domain, but it is computationally inefficient. Common practice is to perturb the displacements about a steady- state configuration by writing,

$$
\begin{aligned}
& \{\mathrm{u}(\mathrm{t})\}=\left\{\mathrm{u}_{0}\right\}+\{\Delta \mathrm{u}(\mathrm{t})\} \\
& \{\mathrm{P}(\{\mathrm{u}\}, \mathrm{t})\}=\left[\mathrm{P}\left(\left\{\mathrm{u}_{0}\right)\right)\right\}+\left[\Delta \mathrm{P}([\Delta \mathrm{u}\}, \mathrm{t})+\left\{\Delta \mathrm{P}^{\mathrm{w}}(\mathrm{t})\right\}\right. \\
& \{\mathrm{F}(\mathrm{t})]=\left\{\mathrm{F}_{0}\right\}+[\Delta \mathrm{F}(\mathrm{t})]
\end{aligned}
$$

where $\left\{u_{0}\right\},\left\{P\left(\left\{u_{0}\right\}\right)\right)$, and $\left\{F_{0}\right\}$ are the steady state values of $\{\mathrm{u}),\{\mathrm{P}(\{\mathrm{u}\}, \mathrm{t})\}$, and $\{\mathrm{F}(\mathrm{t})\}$, respectively. The quantities $\{\Delta \mathrm{u}(\mathrm{t})\},(\Delta \mathrm{P}((\Delta \mathrm{u}), \mathrm{t})\}$, and $\{\Delta \mathrm{F}(\mathrm{t}))$ are perturbations from $\left[\mathrm{u}_{0}\right\}$, $\left\{P\left(\left\{u_{0}\right\}\right)\right\}$, and $\left\{F_{0}\right\}$, respectively. The perturbed aerodynamic force is split into motion-dependent $\{\Delta \mathrm{P}(\{\Delta \mathrm{u}\}, \mathrm{t})\}$ and motion-independent $\left\{\Delta \mathrm{P}^{\mathrm{w}}(\mathrm{t})\right\}$ parts for convenience. Substituting $(\mathrm{u}(\mathrm{t})\},\{\mathrm{P}(\{\mathrm{u}\}, \mathrm{t})\}$, and $[\mathrm{F}(\mathrm{t})\}$ from $\mathrm{Eq}$. (2) into the nonlinear $\mathrm{Eq}$. (1) yields two sets of equations: one steady-state equation for $\left\{\mathrm{u}_{0}\right\}$ and another for the perturbation variable $\{\Delta u(t)\}$. These are 


$$
\begin{aligned}
& {\left[\left[\mathrm{K}_{\mathrm{s}}\right]+\left[\mathrm{K}\left(\left\{\mathrm{u}_{0}\right)\right)\right]\right\}\left(\mathrm{u}_{0}\right\}=\left\{\mathrm{P}\left(\left(\mathrm{u}_{0}\right)\right)\right\}+\left(\mathrm{F}_{0}\right\}} \\
& {[\mathrm{M}]\{\Delta \ddot{u}(\mathrm{t})\}+\left[\left[\mathrm{K}_{\mathrm{s}}\right]+\left[\mathrm{K}\left(\left(\mathrm{u}_{0}\right)\right)\right]\right]\{(\Delta \mathrm{u}(\mathrm{t})\}} \\
& \quad=\{\Delta \mathrm{P}((\Delta \mathrm{u}), \mathrm{t})]+\left\{\Delta \mathrm{P}^{\mathrm{w}}(\mathrm{t})\right\}+(\Delta \mathrm{F}(\mathrm{t})\}
\end{aligned}
$$

The steady-state configuration for a given rotational speed and Mach number is obtained by solving the nonlinear Eq. (3). The stiffness matrix $\left[\mathrm{K}\left(\left[\mathrm{u}_{0}\right)\right)\right]$ includes elastic stiffness and differential stiffness due to centrifugal stiffening loads and steady-state aerodynamic loads. Once the steady-state deflection and the effective total stiffness are known from Eq. (3), the natural frequencies and mode shapes are calculated by solving

$$
[M]\{\Delta \ddot{u}(t)\}+\left[\left[K_{g}\right]+\left[K\left(\left\{u_{0}\right\}\right)\right]\right]\{(\Delta u(t)\}=0
$$

which leads to the generalized mass matrix $\left[\mathrm{M}_{\mathrm{g}}\right]$, the modal matrix $[\phi]$, and the modal frequencies $\omega_{j}$.

\section{Flutter and Forced Response by the Modal Method}

The general vibratory motion can be expressed as a superposition of the contributions of the various normal modes:

$$
\{\Delta u(t)\}=[\phi]\{q(t)\}
$$

Substituting Eq. (6) into Eq. (4) and post-multiplying the result by $[\phi]^{\mathrm{T}}$ leads to

$$
\left[M_{g}\right](\ddot{q})+\left[K_{g}\right]\{q)=[A]\{q\}+(G(t)\}
$$

where

$$
\begin{aligned}
& {\left[\mathrm{M}_{\mathrm{g}}\right]=[\phi]^{\mathrm{T}}[\mathrm{M}][\phi]} \\
& \mathrm{K}_{\mathrm{gjj}}=\mathrm{M}_{\mathrm{gjj}} \omega_{\mathrm{j}}{ }^{2}\left(1+2 \mathrm{i} \zeta_{\mathrm{j}}\right) \\
& {[\mathrm{A}]\{\mathrm{q}\}=[\phi]^{\mathrm{T}}\{\Delta \mathrm{P}(\langle\Delta \mathrm{u}\}, \mathrm{t})\}} \\
& \{\mathrm{G}(\mathrm{t})]=[\phi]^{\mathrm{T}}\left[(\Delta \mathrm{F}(\mathrm{t})\}+\left\{\Delta \mathrm{P}^{\mathrm{w}}(\mathrm{t})\right\}\right]
\end{aligned}
$$

The order of Eq. (7) depends on the number of modes included in Eq. (6). This number is determined by performing numerical experiments. The structural damping in each mode is introduced through the damping ratio $\zeta_{j}$ where the modal index is $j=1,2,3, \ldots, \mathrm{m}$.

Assuming that the motion is simple harmonic at flutter, the generalized coordinate vector of the blade can be written as

$$
\{q\}=\left\{q_{0}\right\} e^{i \omega t}
$$

For a tuned rotor, in which all the blades are identical, the aeroelastic modes consist of the $\mathrm{N}$ individual blades vibrating with equal amplitudes with a fixed interblade phase angle between adjacent blades (Ref. 8). For the $\mathrm{s}^{\text {th }}$ blade vibrating in the $\mathrm{r}^{\text {th }}$ interblade phase angle mode, Eq. (9) can be written as

$$
\{q\}=\left\{q_{0}\right\} e^{i\left(\omega t+\sigma_{r} s\right)}
$$

where the phase angle between adjacent blades is given as

$$
\sigma_{r}=2 \pi r / N \quad r=0,1,2, \ldots, N-1
$$

In Eq. (8), the generalized aerodynamic matrix is represented by $[A]$ for each interblade phase angle for simple harmonic motion of the blade. This matrix is defined in Appendix A. The motion-independent aerodynamic and nonaerodynamic forces are represented by $\{G(t)\}$ for each interblade phase angle. These forces are included for completeness in the formulation, but no results on forced response will be presented in this paper. After setting $\{G(t)\}=\{0\}$ and substituting Eq. (10), Eq. (7) becomes

$$
-\omega^{2}\left[M_{g}\right]\left\{q_{0}\right\}+\left[K_{g}\right]\left\{q_{0}\right\}=[A]\left\{q_{0}\right\}
$$

Simplifying further, the flutter eigenvalue problem can be written as

where

$$
[P]\left\{q_{o}\right\}=\gamma\left[M_{g}\right]\{q\}
$$

$$
\begin{aligned}
& {[P]=\left[K_{g}\right]-[A]} \\
& i \sqrt{\gamma}=i \omega=\mu+i v
\end{aligned}
$$

Flutter occurs when $\mu>0$. $\omega$ is the flutter frequency and $\gamma$ is the eigenvalue obtained from the solution of Eq. (13).

\section{ASTROP2 Computer Code}

A detailed description of the ASTROP2 code can be found in Refs. 1 and 6. The ASTROP2 code is based on a fully coupled normal mode structural model and $2 \mathrm{D}$ unsteady cascade aerodynamic theory.

Four external datasets must be furnished to ASTROP2 for an analysis condition. These datasets define: (1) the steady aerodynamic inflow into each rotor, (2) the blade steady state geometry, (3) the blade dynamic characteristics in the form of mode shapes and frequencies, and (4) the blade setting angle and freestream aerodynamic information. In the present analysis, the aerodynamic solver of Ref. 9 was used to provide the nonuniform steady inflow (item(1)) into each rotor, and MSC/NASTRAN (Ref. 10) was used to provide items (2) and (3) as described below. Item (4) was user input to ASTROP2 directly.

ASTROP2 combines the normal modes calculated by NASTRAN and a 2D-aerodynamic strip representation of the blade to calculate the generalized aerodynamic matrix, [A], described in Appendix A. It uses a frequency domain solution which predicts frequency, damping, and phase angle of aeroelastic modes. It is a single rotor analysis, meaning no rotor-to-rotor unsteady aerodynamics or structural dynamic interaction is accounted for. However, steady aerodynamic interaction between rotors can be accounted for by user input of the steady flowfield into each rotor. This is the state of the art technology for aeroelastic analysis of $\mathrm{CR}$ propellers. 
ASTROP2 computes the unsteady aerodynamic loads (using the theory presented in Refs. 11 and 12.) and the damping and frequency for each respective interblade phase angle of the aeroelastically coupled modes.

\section{Code Modifications}

Previously, the ASTROP2 code modeled single rotation propfans with a uniform steady inflow velocity distribution, and the analysis was manually iterated to converge upon the least stable mode. Additions and modifications have been made to model each rotor of counterrotation propfans (neglecting structural and unsteady aerodynamic rotor-to-rotor interaction) and to automatically converge upon the least stable mode. The finite element geometry and corresponding modal deflection information for the aft blades are transformed to simulate the forward blades. In addition, the axial and circumferential nonuniform steady flow into each rotor can be input to include the rotor-to-rotor steady aerodynamic interaction effects. This nonuniform inflow velocity information is used to calculate the local relative Mach number for each aerodynamic strip and the unsteady aerodynamic loads. For a counterrotating configuration, the local Mach number distribution changes significantly due to aerodynamic interactions between the rotors and affects the unsteady aerodynamic loads. For single rotation configurations it is sufficient to assume uniform inflow velocity. A separate steady aerodynamic solver (Ref. 9) was used to provide the nonuniform steady inflow velocity information in the present analysis. For the blade designs analyzed in this paper, the use of a nonuniform inflow velocity distribution provided less conservative stability results than would have been obtained from a uniform inflow velocity.

The aeroelastic stability analysis was modified to automatically iterate (to converge) and identify the least stable (damped) mode and the corresponding interblade phase angle for the rotor being analyzed. The procedure used is as follows. First, a value for blade vibration frequency is specified by the user. Using this value, ASTROP2 calculates the unsteady aerodynamic loads and aeroelastic eigenvalues for all the interblade phase angle modes. Based on the damping, the least stable mode is then identified. The frequency associated with this mode is then used to calculate new unsteady aerodynamic loads and aeroelastic eigenvalues. This procedure is repeated automatically till the frequency used to calculate the unsteady aerodynamic loads and the frequency associated with the least damped aeroelastic mode are the same. This routine saves a significant amount of time compared to the former manual method used. This is especially true when the relative tip Mach number exceeds 1 and convergence is slower. Also, the automatic iteration is useful in analyzing a large number of operating conditions as was required in the present study.
Formerly, a Newton-Cotes method was used to integrate the unsteady aerodynamic forces on spanwise strips to calculate the total lift and moment on the blade. The strip layout scheme in ASTROP2 lays strips normal to the blade leading edge. For propfan blades with varying sweep along the span, this scheme generates strips of unequal spanwise width. However, the Newton-Cotes method is only valid for strips of equal width and large errors are introduced when analyzing swept propfan blades with strips of unequal spanwise width. Therefore, an alternative integration method was needed. For increased accuracy in the ASTROP2 code, a trapezoidal integration scheme has been implemented to calculate the unsteady aerodynamic forces on blades. The trapezoidal integration method is valid for strips of unequal width and gives accurate results for swept propfan blades. Analyses using the modified code have been verified with existing propfan model wind tunnel flutter data from Ref. 1.

\section{Structural Model and Blade Geometry}

Blade geometry and material information are first input to a composite material and geometric preprocessor for NASTRAN, called COBSTRAN (Ref. 13). COBSTRAN produces grid mesh, element geometry, and material properties in the form of NASTRAN bulk data cards. MSC/NASTRAN then provides both: a geometric nonlinear structural analysis to calculate the blade steady-state deformed configuration and total differential stiffness; and a complete vibration analysis which calculates the natural frequencies and mode shapes of the blade deformed state.

\section{Blade Description and Analysis Conditions}

The propfan models were designed for tests in the NASA Ames $14 \mathrm{ft}$. wind tunnel. The two blade sets under analysis were the primary blades for the program. The CM1 blade set represents a geared propeller design and the CM2 blade set an ungeared propeller design. CM1 blades are of higher aspect ratio than the CM2 blades and the design tip speed of the CM1 blades is lower than that of the CM2 blades (see Table 1). The hub diameter of the rotors is $0.216 \mathrm{~m}$ (8.5 in.). The design total thrust for the model blades at cruise RPM (two counterrotating blade rows, six blades per row $)$ is $622.7 \mathrm{~N}(140 \mathrm{lbs})$. All the blades are made from layered pre-impregnated graphite composite material $0.08 \mathrm{~mm}(0.0032 \mathrm{in})$ thick, with 90 plies in each CM1 blade and 82 plies in each CM2 blade.

The finite element models for the CM1 and CM2 forward and aft blades are shown in Figures 2 and 3. A rigidly clamped connection was assumed at the blade shank end. The hub that retained each blade set was assumed rigid and was not modeled.

The blade flutter analyses conditions for the CM1 and CM2 blades are shown in Table 2. For both blade designs 
there were three tip speeds analyzed at each of three Mach numbers. In addition, the CM2 blades were analyzed at a fourth Mach number at some conditions. Also, the analysis at each operating condition was done for the first six natural modes of each of the four blade designs. Hence, more than 216 conditions were analyzed for each of the final blade designs. During the design process three other CM1 and four other CM2 designs were considered. Most of these designs were also analyzed over the full set of analyses conditions.

\section{Results and Discussion}

The calculated results for the CM1 and CM2 blade sets are presented in two parts: (1) the in vacuum blade natural frequency variation with rotational speed, and the mode shapes at the design rotational speed; (2) calculated aeroelastic damping plots, damping versus axial Mach number. In part (1) above, the calculations were made using MSC/NASTRAN. The calculations of blade steady state deflected positions included centrifugal loads but not steady airloads. For both the CM1 and CM2 blade designs, the steady airloads were significantly smaller than the centrifugal loads. It was shown in Ref. 1 that the steady airloads do not have a significant effect on the natural frequencies. The real and imaginary parts of the eigenvalue for part (2) above were calculated using ASTROP2. Usually, $0.5 \%$ or greater structural damping is present in composite blades, but zero structural damping was assumed in the present analysis. This assumption makes the calculated stability results conservative. Six natural modes for each blade were included in the analysis although only results for the three least stable aeroelastic modes are presented here.

\section{Blade Natural Frequencies and Mode Shapes}

Figures 4 and 5 show the calculated variation of the first three natural frequencies with increasing rotational speed in vacuum for the $\mathrm{CM} 1$ and $\mathrm{CM} 2$ forward and aft blades. The modal frequencies increase with an increase in rotational speed. This trend is expected because of centrifugal stiffening.

For the CM1 blades, the percent stiffening from $0 \mathrm{~m} / \mathrm{s}$ blade tip speed to $228.6 \mathrm{~m} / \mathrm{s}(750 \mathrm{ft} / \mathrm{s})$ is calculated for the modes shown in Figure 4. For the forward blades, seen in Figure 4(a), the first mode shows the maximum percent stiffening at $10.8 \%$ and the third mode shows the minimum at $2.5 \%$. For the aft blades, seen in Figure $4(\mathrm{~b})$, the first mode shows the maximum percent stiffening at $12.3 \%$ and the third mode shows the minimum at $3.9 \%$.

For the CM2 blades, the percent stiffening from $0 \mathrm{~m} / \mathrm{s}$ blade tip speed to $274.3 \mathrm{~m} / \mathrm{s}(900 \mathrm{ft} / \mathrm{s})$ is calculated for the modes shown in Figure 5. For the forward blades, seen in Figure 5(a), the first mode shows the maximum percent stiffening at $15.5 \%$ and the third mode shows the minimum at $2.9 \%$. For the aft blades, seen in Figure 5(b), the first mode shows the maximum percent stiffening at $15 \%$ and the third mode shows the minimum at $1.8 \%$.

Also shown in Figures 4 and 5 are the ranges of bench measured natural frequencies of the manufactured blades. For both the forward and aft blades, there were 26 blades measured using laser speckle interferometry. It can be seen that the measured natural frequencies are typically lower than calculated. The effect of these lower blade natural frequencies on calculated damping will be discussed in the next section.

Figures 6 and 7 show the calculated iso-displacement contours of the CM1 and CM2 blades for the first six natural modes at the design rotational speed. Note that all the modes of CM1 and CM2 consist of coupled bending, torsion and edgewise motions. The modes referred to below are meant to identify the primary motion that can be associated with a single-degree-of-freedom beam mode. For both CM1 blades, the first two modes are first and second bending respectively, and the third mode is torsion. Whereas, for both CM2 blades, the first mode is first bending, the second mode is first torsion, and the higher modes are platelike.

\section{Calculated Damping}

For a six bladed rotor, it is theoretically possible for the rotor to go unstable in six interblade phase angle modes for each of the six normal modes included in the analysis. Only the most critical interblade phase angle mode (the one closest to instability) is shown here for each of the three least stable normal modes. The unsteady aerodynamic loads were calculated using twenty chordwise strips across the blade span.

Figure 8 shows the variation of the aeroelastic damping coefficient (the real part of the eigenvalue) with freestream Mach number for the CM1 forward blades at three different blade tip speeds. The three least stable modes are shown. Figure $8(\mathbf{c})$ is discussed here in detail as it corresponds to the maximum tip speed analyzed for the CM1 forward blade. In Figure 8(c), the interblade phase angles corresponding to the least stable modes are: first, $180^{\circ}$; second, $60^{\circ}$; and fourth, $300^{\circ}$. A blade was considered to be flutter free when the aerodynamic damping was more than 0.002 in all the modes. From Figure 8 , it can be seen that over the entire operating range, the CM1 forward blades are flutter free.

Figure 9 shows the variation of the aeroelastic damping coefficient with freestream Mach number for the CM1 aft blades at three different blade tip speeds. The three least stable modes are shown. For the maximum tip speed analyzed, seen in Figure $9(\mathrm{c})$, the interblade phase angles corresponding to the least stable modes are: first, $180^{\circ}$; second, $0^{\circ}$; and fourth, $300^{\circ}$. From Figure 9 , it can be 
seen that over the entire operating range, the CMI aft blades are flutter free.

Figure 10 shows the variation of the aeroelastic damping coefficient with freestream Mach number for the CM2 forward blades at three different blade tip speeds. The three least stable modes are shown. For the maximum tip speed analyzed, seen in Figure $10(\mathrm{C})$, the interblade phase angles corresponding to the least stable modes are: second, $120^{\circ}$; fifth, $300^{\circ}$; and sixth, $180^{\circ}$. Though the damping in the sixth mode is very small, it is considered aeroelastically stable due to the very high frequency. From Figure 10, it can be seen that over the entire operating range, the CM2 forward blades are flutter free.

Figure 11 shows the variation of the aeroelastic damping with freestream Mach number for the CM2 aft blades at three different blade tip speeds. The three least stable modes are shown. For the maximum tip speed analyzed, seen in Figure 11(c), the interblade phase angles corresponding to the least stable modes are: fourth, $60^{\circ}$; fifth, $300^{\circ}$; and sixth, $240^{\circ}$. From Figure 11 , it can be seen that over the entire operating range, the CM2 aft blades are flutter free.

The results presented in Figures 8-11 were obtained using the calculated blade natural frequencies. As seen in Figures 4 and 5 , the measured blade frequencies were typically lower than the calculated frequency values. To study the effect of these lower frequencies, the analyses were repeated for natural frequencies $10 \%$ lower than the calculated ones. The aerodynamic damping remained the same for most of the modes and increased in some cases. All the blade designs were still predicted to be flutter free within the planned operating range using these lower natural frequencies. However, although aerodynamic damping was not changed much in these blade designs by the lower natural frequencies, in other designs aerodynamic damping may be adversely affected. Hence, each design must be independently checked.

The aeroelastic modal frequencies were very close to the natural frequencies for all these blade designs. This indicates that there was very little aeroelastic coupling between different modes and all the aeroelastic modes were well separated.

In the wind tunnel test, the CM1 and CM2 blade sets did not flutter. This enhances the confidence in the ASTROP2 code as an aeroelastic design tool for both single and counter rotation propfan configurations.

\section{Conclusions}

An aeroelastic stability research code for single rotation propfans was modified and successfully applied to counterrotation propfans.
The code was used to analyze the aeroelastic stability of two different counterrotation propfan blade designs for the joint Navy/Air Force/NASA cruise missile wind tunnel test. Both the blade designs, called CM1 and CM2, were predicted to be flutter free. Experimentally this was confirmed over the entire operating range of the wind tunnel test.

\section{Acknowledgements}

The authors would like to thank Dr. T.S.R. Reddy for his contributions in the development of the version of ASTROP2 code used in this analysis. The authors would also like to thank L. J. Krzycki, Propulsion Systems Division and L. W. Strutz, Missile Airframe Division, Naval Weapons Center, China Lake for supporting this work.

\section{References}

1. Kaza, K.R.V., Mehmed, O., Narayanan, G.V. and Murthy, D.V., "Analytical Flutter Investigation of a Composite Propfan Model," „I Aircraft, Vol.26, No.8, August 1989, pp. 772-780.

2. Strack, W.C., et al, "Technology and Benefits of Aircraft Counter Rotation Propellers," NASA TM-82983, 1981.

3. Groeneweg, J.F. and Bober, L.J., "Advanced Propeller Research, Aeropropulsion '87, Session 5, Subsonic Propulsion Technology," NASA CP-10003-SESS-5, 1987.

4. Henderson, B.W., "Propfan Engine May Be Suitable for Next-Generation Cruise Missile," Aviation Week \& Space Technology, Vol.136, No.1, Jan. 6, 1992, pp. 62-63.

5. Fite, E.B., Miller, C.J., Mehmed, O. and Stefko, G.L., "Overview of Cruise Missile Composite Propfan Design, Analysis and Fabrication," NASA TM-105264, to be published.

6. Narayanan, G.V. and Kaza, K.R.V., "ASTROP2 User's Manual: A Program For Aeroelastic Stability Analysis of Propfans," NASA TM-4304, August 1991.

7. Subrahmanyam, K.B., Kaza, K.R.V., Brown, G.V. and Lawrence, C., "Nonlinear Bending-Torsional Vibration and Stability of Rotating, Pretwisted, Preconed Blades Including Coriolis Affects," NASA TM-87207, 1986.

8. Lane, F., "System Mode Shapes in the Flutter of Compressible Blade Rows," J. Aeronaut. Sci., Vol. 23, No. 1, Jan. 1956, pp. 54-66.

9. Miller, C.J., "Aerodynamic Design of Aft-Mounted Counter Rotating Propfans for a Cruise Missile Model," NASA TM-105266, to be published.

10. The MSC/NASTRAN Theoretical Manual, NASA SP. $221(06), 1981$. 
11. Smith, S.N., "Discrete Frequency Sound Generation in Axial Flow Turbomachines," ARC-R\&M-3709, British ARC, 1973.

12. Adamczyck, J.J. and Goldstein, M.E., "Unsteady Flow in a Supersonic Cascade with Subsonic Leading-Edge Locus," AIAA J., Vol. 16, No. 12, pp. 1248-1254, December 1978

13. Aiello, R.A., "Composite Blade Structural Analyzer (COBSTRAN) User's Manual," NASA TM-101461,1989.

\section{Appendix A}

\section{Calculation of Generalized Aerodynamic Forces}

It is assumed that the lift and moment per unit span due to blade motion are linearly related to the displacements and their derivatives with respect to arc length along a reference line. The blade is divided into a series of discrete aerodynamic strips. Each strip has two motions, pitching $(\alpha)$ and plunging (h) about an arbitrary reference line. The lift and moment expressions per unit span are

$$
\left\{\begin{array}{r}
L \\
M_{a}
\end{array}\right\}=\pi \rho \omega^{2}\left[\begin{array}{llll}
l_{11} & l_{12} & \ldots & l_{16} \\
l_{21} & l_{22} & \ldots & l_{26}
\end{array}\right]\left\{\begin{array}{c}
h \\
\alpha \\
h^{\prime} \\
\alpha^{\prime} \\
h^{\prime \prime} \\
\alpha^{\prime \prime}
\end{array}\right\}
$$

The prime denotes the differentiation with respect to the arc length along the reference line. The elements $l_{11}, l_{12}$, etc, are defined in Ref. 1. The pitching and plunging displacements and their derivatives are given by

$$
\left|\begin{array}{c}
h \\
\alpha \\
h^{\prime} \\
\alpha^{\prime} \\
h^{\prime \prime} \\
\alpha^{\prime \prime}
\end{array}\right|=\left\{\begin{array}{l}
{\left[w^{\prime}\right]} \\
{\left[w^{\prime}\right]} \\
{\left[W^{\prime \prime}\right]}
\end{array}\right\}\{q\}
$$

where

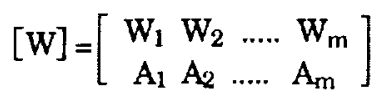

Here $W_{j}$ and $A_{j}, j=1, \ldots, m$, are the modal displacement amplitudes and rotations, respectively, and $m$ is the number of normal modes included in the analysis.

The generalized aerodynamic force vector can be expressed in terms of lift, moment, normal modes, and normal coordinates as

$$
\{\mathrm{Q}\}=\pi \rho \omega^{2} \int_{0}^{1}[\mathrm{~W}]^{\mathrm{T}}\left[\begin{array}{c}
\mathrm{L} \\
\mathrm{M}_{\mathrm{a}}
\end{array}\right] \mathrm{ds}=[\mathrm{A}]\{\mathrm{q}\}
$$

where 1 represents the length of the reference axis. Substituting Eqs. (A1) and (A2) into Eq. (A4),

$$
[\mathrm{A}]=\pi \rho \omega^{2} \int_{0}^{1}[\mathrm{~W}]^{\mathrm{T}}[\mathrm{LCOEF}]\left\{\begin{array}{l}
{[\mathrm{W}]} \\
{\left[\mathrm{W}^{\prime}\right]} \\
{\left[\mathrm{W}^{\prime \prime}\right]}
\end{array}\right\} \mathrm{ds}
$$

where

$$
[\mathrm{LCOEF}]=\left[\begin{array}{llll}
l_{11} & l_{12} & \ldots & l_{16} \\
l_{21} & l_{22} & \ldots & l_{26}
\end{array}\right]
$$

\begin{tabular}{|c|c|c|}
\hline & CM1 & CM2 \\
\hline $\begin{array}{c}\text { Freestream Mach } \\
\text { number }\end{array}$ & 0.4 to 0.85 & 0.4 to 0.9 \\
\hline $\begin{array}{c}\text { Max. Rotor Speeds } \\
\text { rpm }\end{array}$ & 10417 & 14475 \\
\hline $\begin{array}{c}\text { Tip Diameter } \\
\text { m (in) }\end{array}$ & $0.425(16.75)$ & $0.368(14.5)$ \\
\hline $\begin{array}{c}\text { Design Conditions } \\
\text { Mach number } \\
\text { tip speed, m/s (ft/s) } \\
\text { rpm } \\
\text { blade angle, 3/4R, } \\
\text { forward blade } \\
\text { aft blade }\end{array}$ & $213.4(700)$ & $274.3(900)$ \\
& 9580 & 14225 \\
\hline \hline
\end{tabular}

TABLE 1 - BLADE DESIGN CONDITIONS

\begin{tabular}{|c|c|c|}
\hline Blade & Mach Numbers & Tip Speeds, m/s (ft/s) \\
\hline \hline CM1 Forward and CM1 Aft & $0.4,0.7,0.85$ & $137.2(450), 182.9(600), 228.6(750)$ \\
\hline CM2 Forward and CM2 Aft & $0.4,0.6,0.8,0.9$ & $182.9(600), 228.6(750), 274.3(900)$ \\
\hline
\end{tabular}

TABLE 2 - BLADE FLUTTER ANALYSES CONDITIONS 


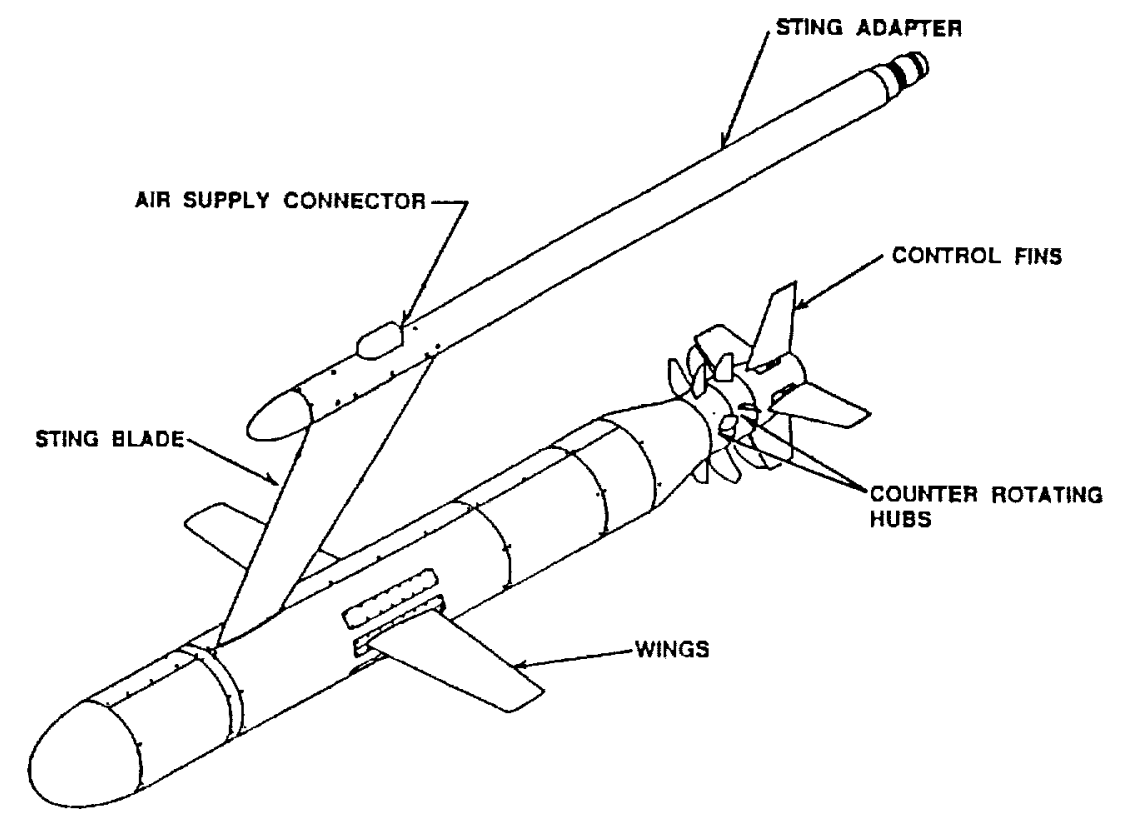

FigURE I Propfan Missile Interactions Model

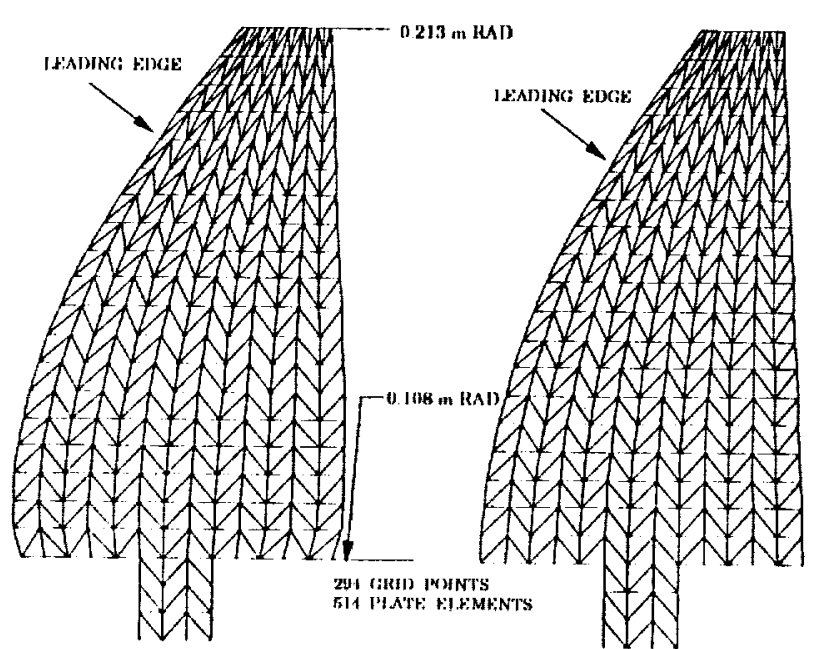

(a) Forward Blade

(b) An Blade

Fig. 2 Finite Element Models for CM1 Blade Set

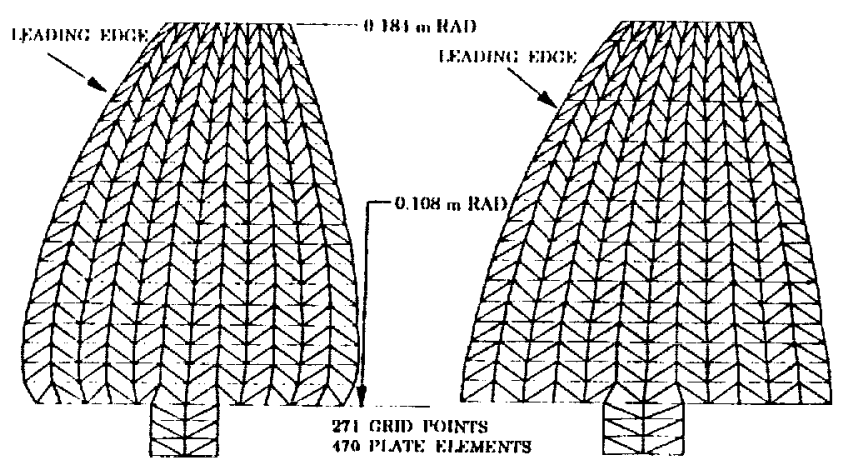

(a) Forward Blade

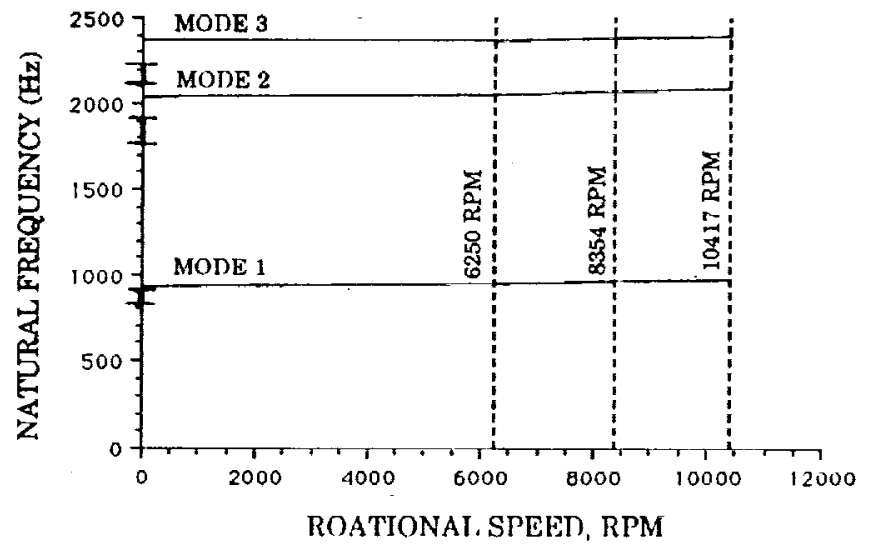

(a) Forward Blade

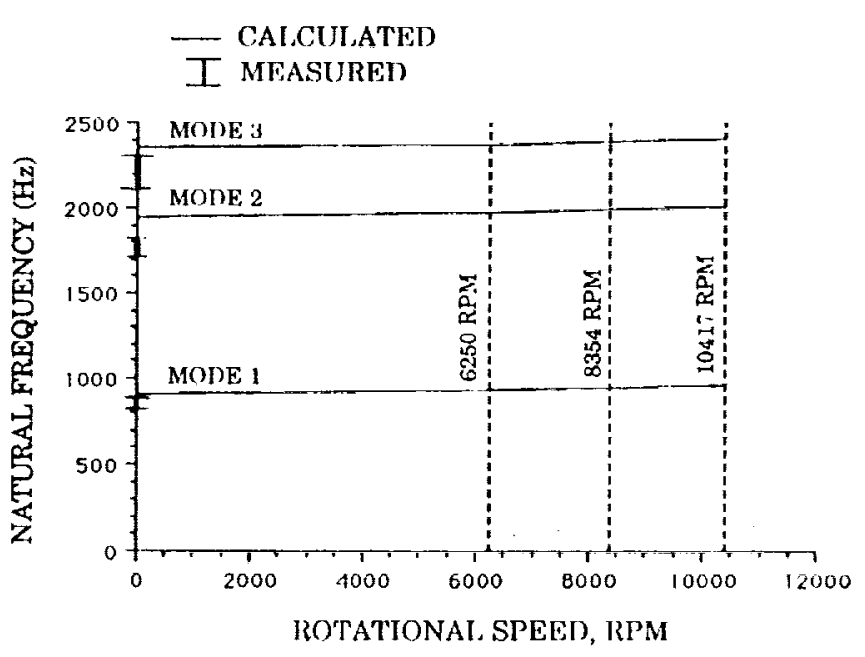

(b) Aft Blade

Figure 4 CM 1 - Variation of natural frequency with rotational speed 


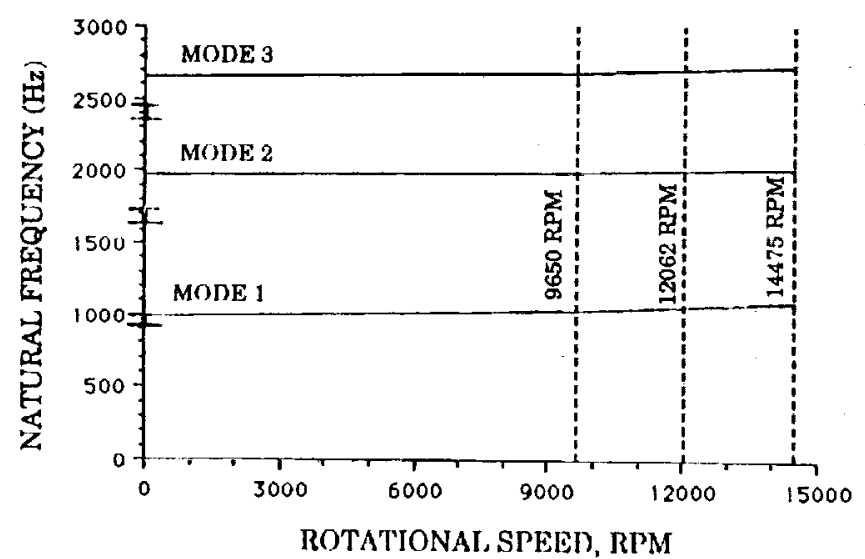

(a) Forward Blade

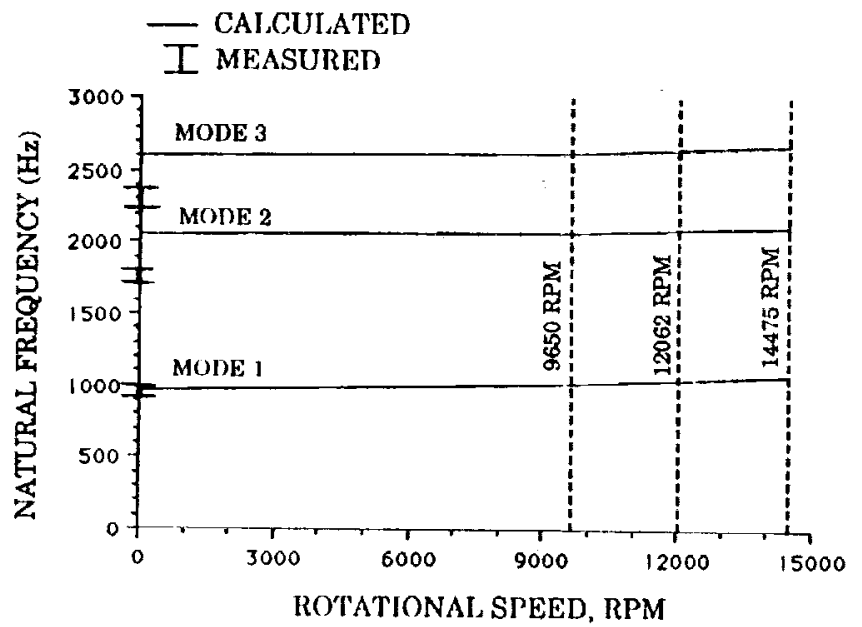

(b) Aft Blade

Figure 5 CM2 - Variation of natural frequency with rotational speed
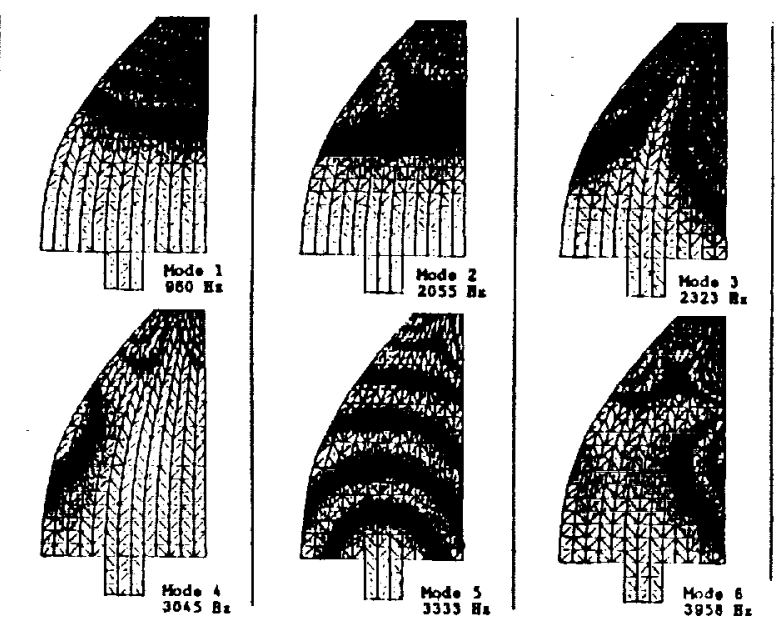

(a) Forward Blade
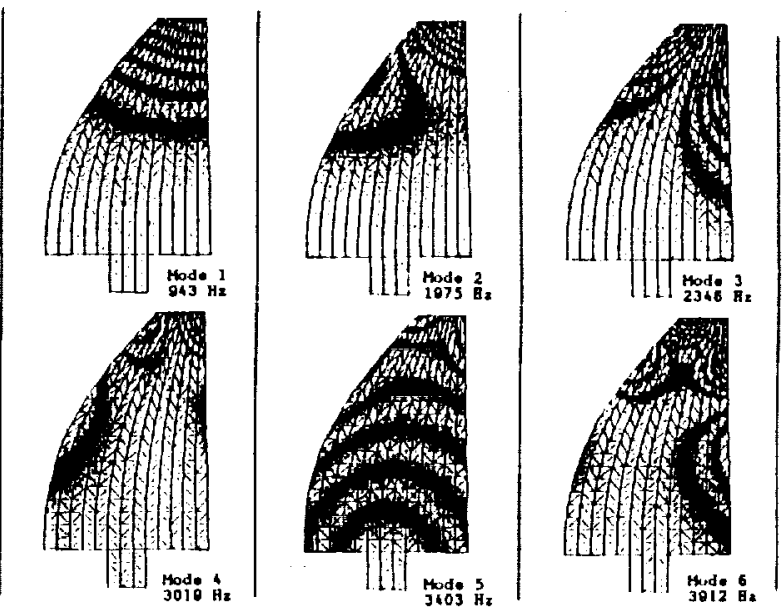

(b) Aft Blade

Figure 6 CM1 Mode Shapes at $228.6 \mathrm{~m} / \mathrm{s}(750 \mathrm{tt} / \mathrm{s})$

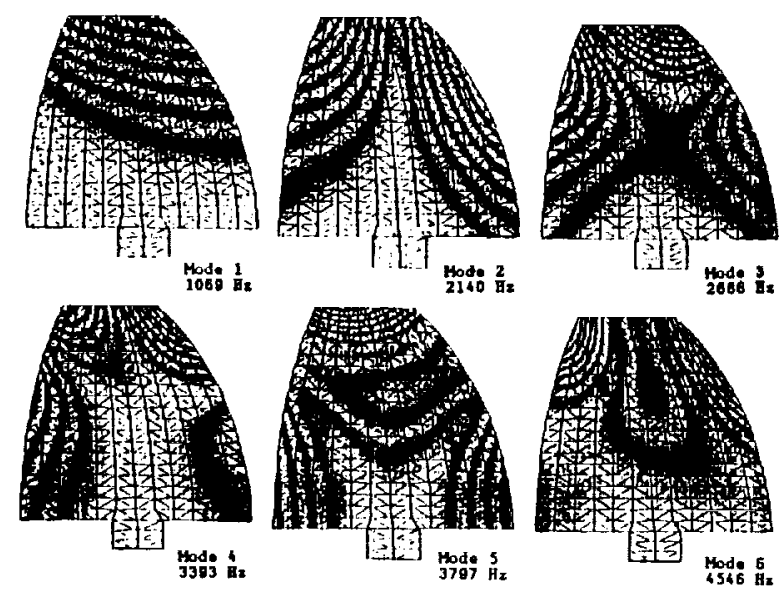

(a) Forward Blade
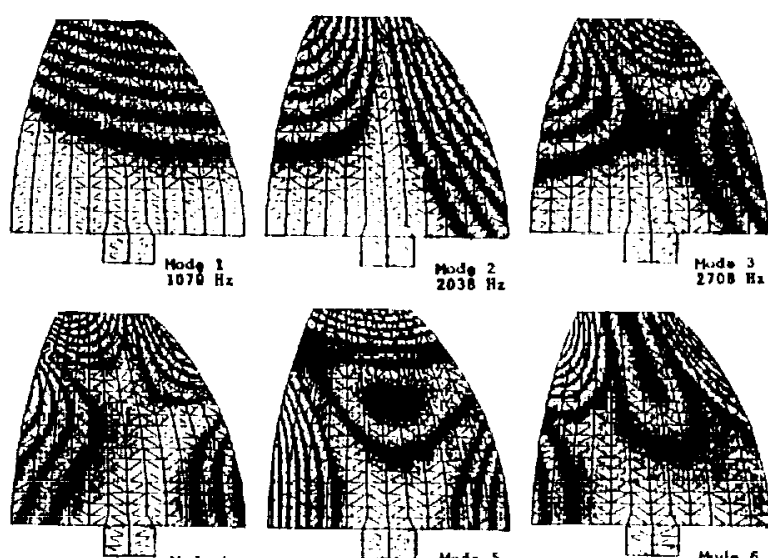

tod.

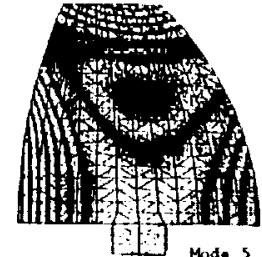

${ }_{4059}^{\text {Modi }} \mathrm{Hz}^{5}$

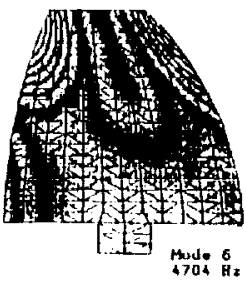

(b) Aft Blade

Figure 7 CM2 Mode Shapes at $274.3 \mathrm{~m} / \mathrm{s}(900 \mathrm{ft} / \mathrm{s})$ 


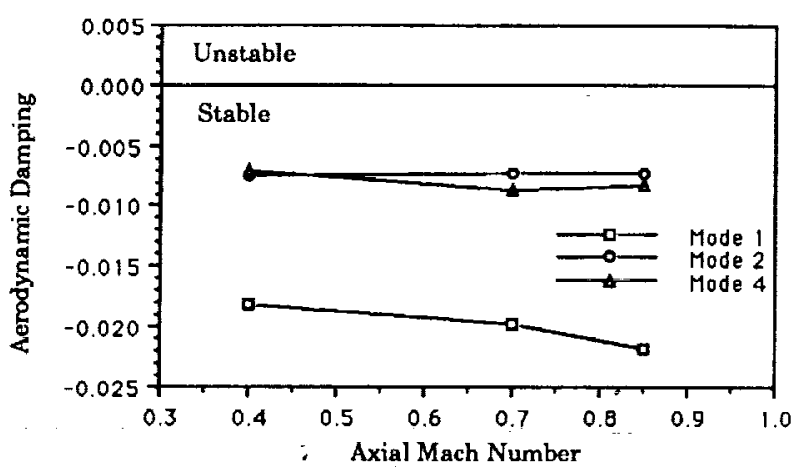

(a)

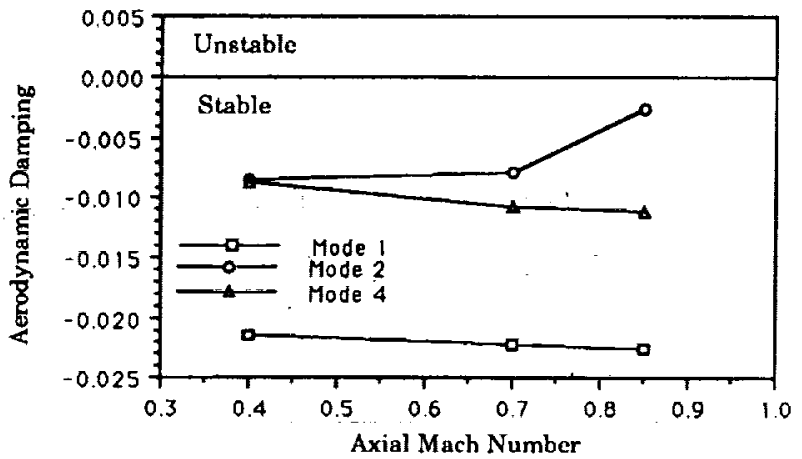

(b)

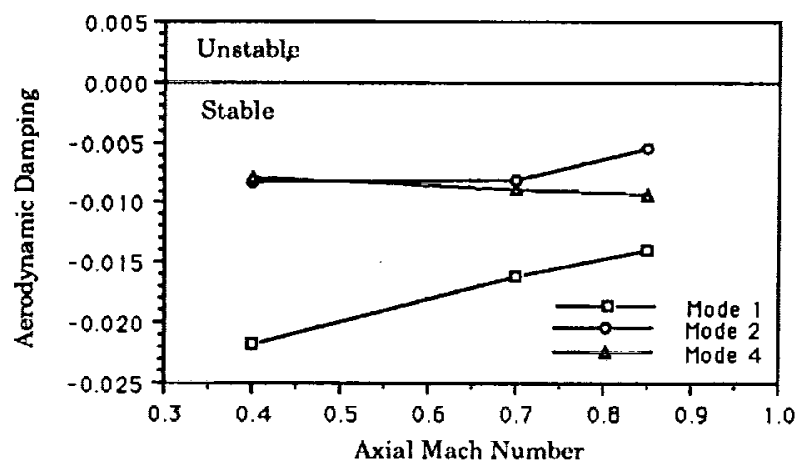

(c)

Figure 8 Variation of damping with Mach number and blade tip speed for CM1 forward blade (a) $137.2 \mathrm{~m} / \mathrm{s}(450 \mathrm{ft} / \mathrm{s})$ (b) $182.9 \mathrm{~m} / \mathrm{s}(600 \mathrm{ft} / \mathrm{s})$ (c) $228.6 \mathrm{~m} / \mathrm{s}(750 \mathrm{ft} / \mathrm{s})$

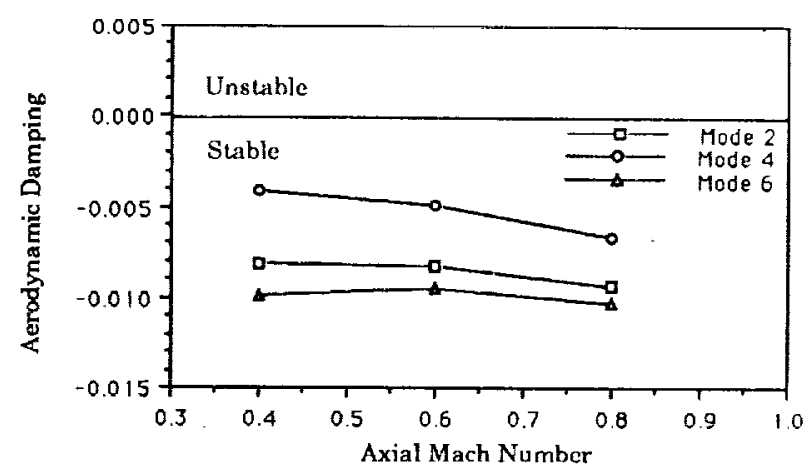

(a)

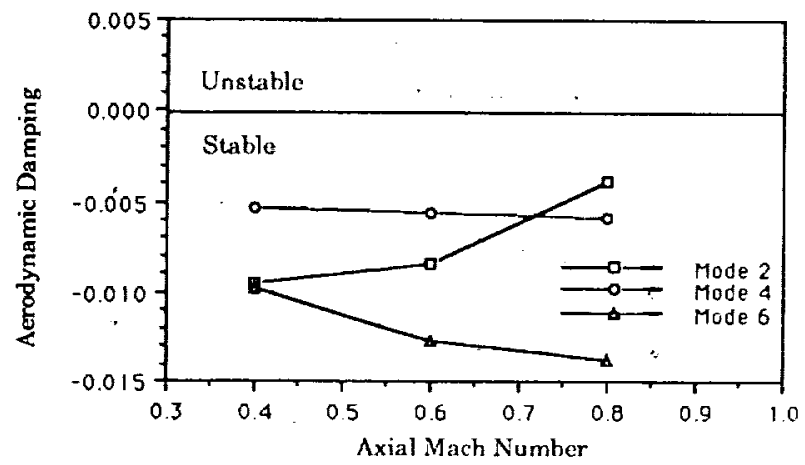

(b)

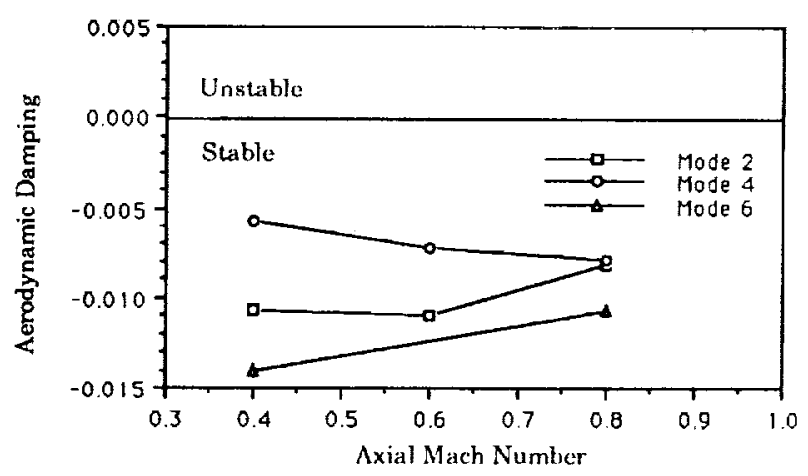

(c)

Figure 9 Variation of damping with Mach number and blade tip speed for CM1 aft blade (a) $137.2 \mathrm{~m} / \mathrm{s}(450 \mathrm{ft} / \mathrm{s})$ (b) $182.9 \mathrm{~m} / \mathrm{s}(600 \mathrm{ft} / \mathrm{s})(\mathrm{c}) 228.6 \mathrm{~m} / \mathrm{s}(750 \mathrm{ft} / \mathrm{s})$ 


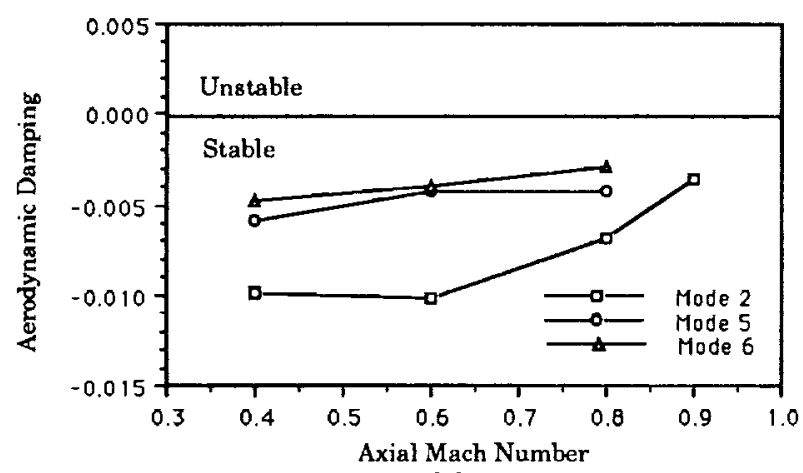

(a)

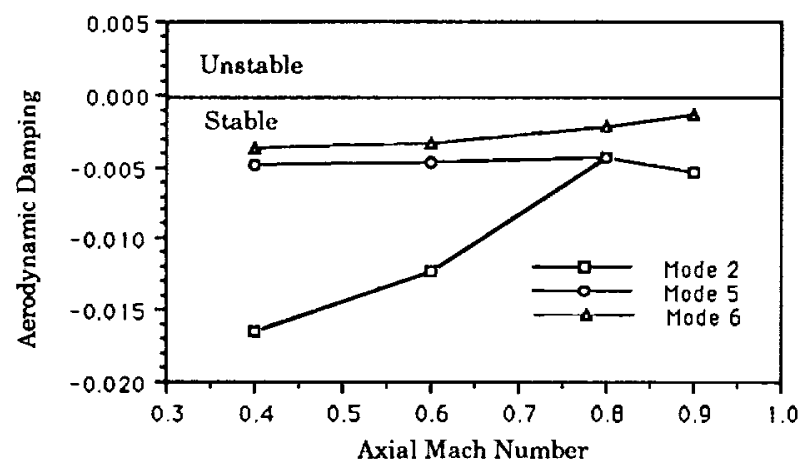

(b)

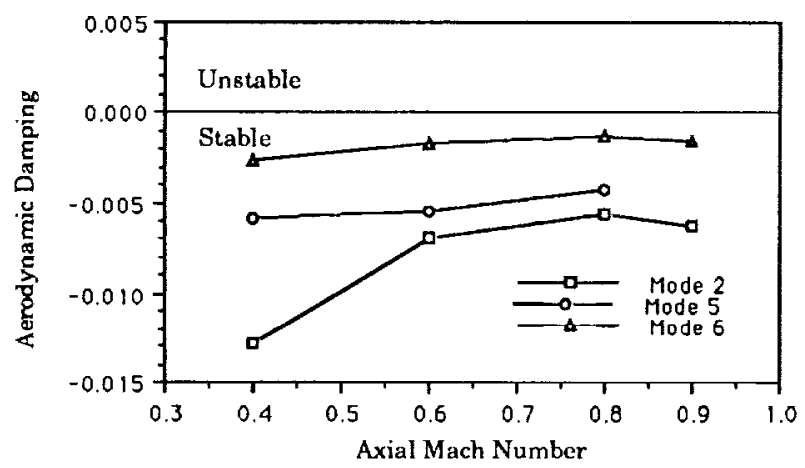

(c)

Figure 10 Variation of damping with Mach number and blade tip spced for CM2 forward blade (a) $182.9 \mathrm{~m} / \mathrm{s}(600 \mathrm{ft} / \mathrm{s})$ (b) $228.6 \mathrm{~m} / \mathrm{s}(750 \mathrm{ft} / \mathrm{s})$ (c) $274.3 \mathrm{~m} / \mathrm{s}(900 \mathrm{ft} / \mathrm{s})$

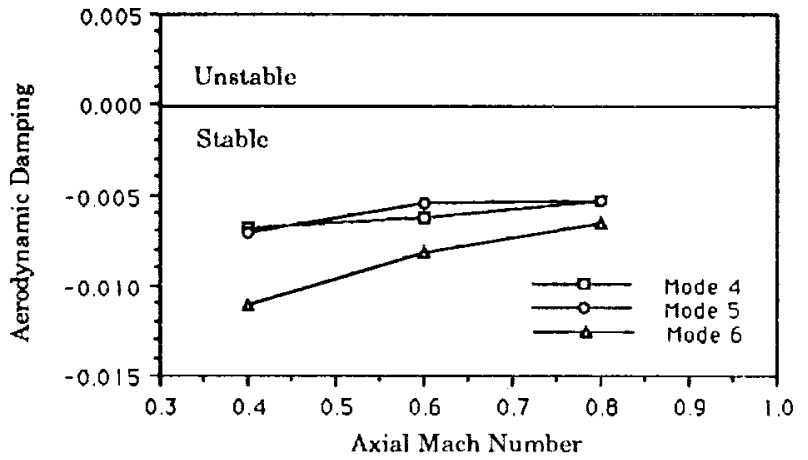

(a)

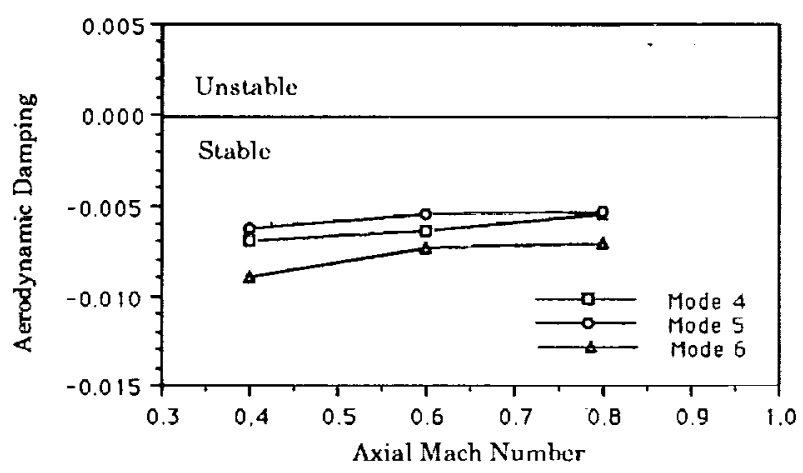

(b)

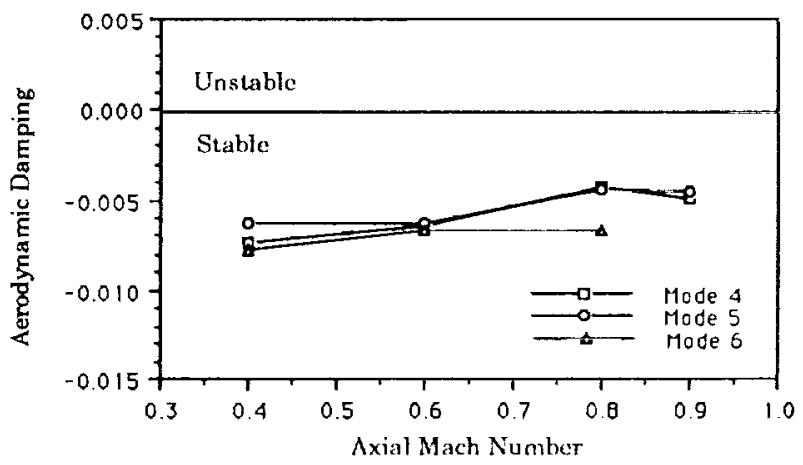

(c)

Figure 11 Variation of damping with Mach number and blade tip speed for CM2 aft blade (a) $182.9 \mathrm{~m} / \mathrm{s}(600 \mathrm{ft} / \mathrm{s})$ (b) $228.6 \mathrm{~m} / \mathrm{s}(750 \mathrm{ft} / \mathrm{s})(\mathrm{c}) 274.3 \mathrm{~m} / \mathrm{s}(900 \mathrm{ft} / \mathrm{s})$ 


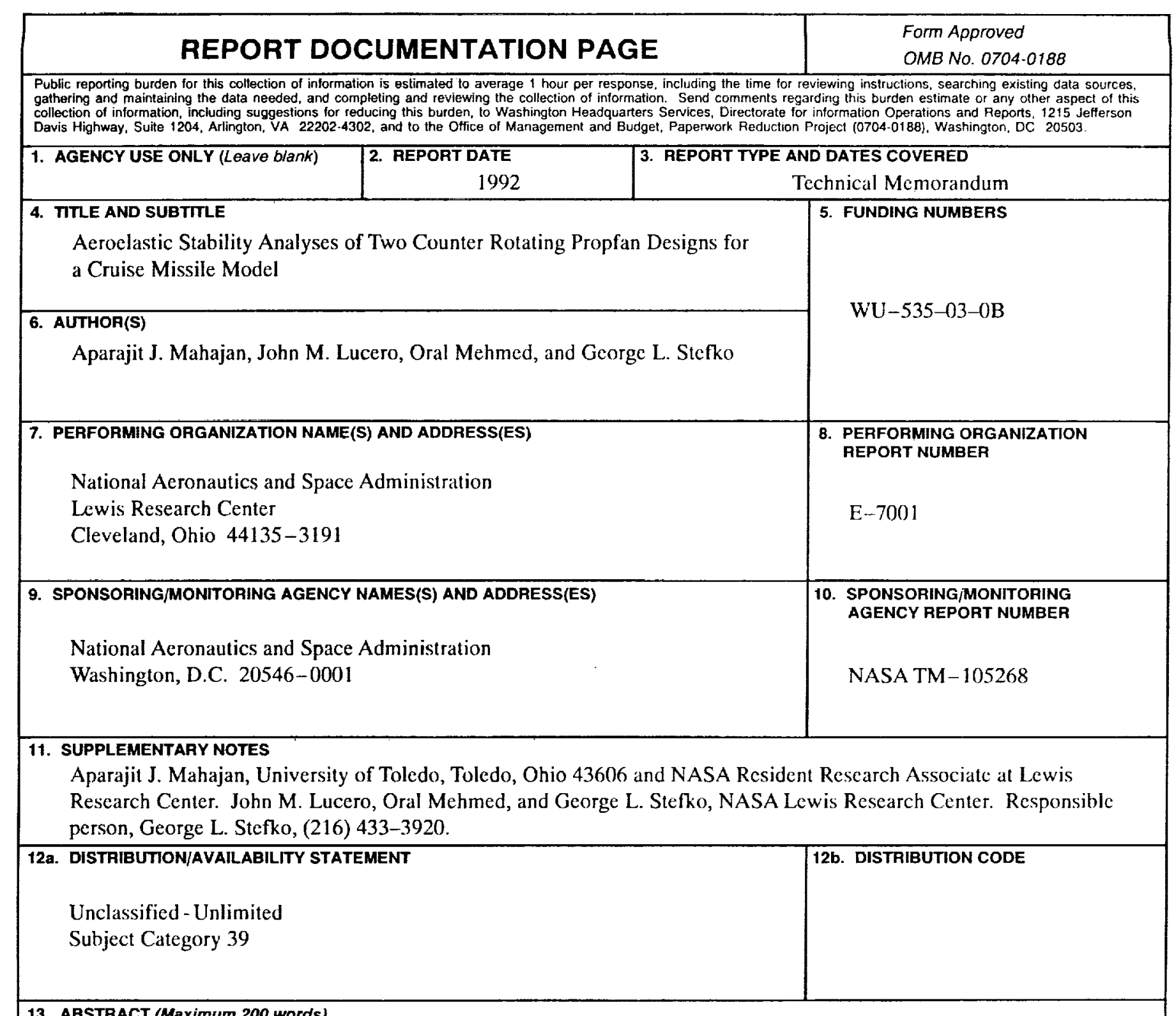

13. ABSTRACT (Maximum 200 words)

Aeroelastic stability analyses were performed to insure structural integrity of two counterrotating propfan blade designs for a NAVY/Air Force/NASA cruise missile model wind tunnel test. This analysis predicted if the propfan designs would be flutter free at the operating conditions of the wind tunnel test. Calculated stability results are presented for the two blade designs with rotational speed and freestream Mach number as parameters. An acroclastic analysis code ASTROP2 (Aeroelastic STability and Response Of Propulsion Systems - 2 Dimensional Analysis), developed at LeRC, was used in this project. The aeroelastic analysis is a modal method and uses the combination of a finite element structural model and two dimensional steady and unsteady cascade aerodynamic models. This code was developed to analyze single rotation propfans but was modified and applied to counterrotation propfans for the present work. Modifications were made to transform the geometry and rotation of the aft rotor to the same reference frame as the forward rotor, to input a non-uniform inflow into the rotor being analyzed, and to automatically converge to the least stable acroelastic mode.

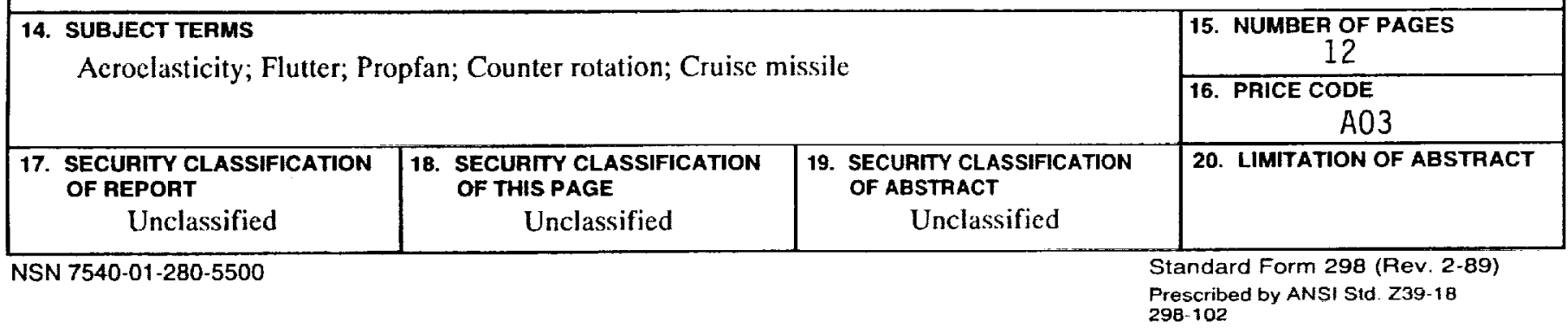

